\title{
Hidden QCD in Chiral Gauge Theories
}

\author{
Thomas A. RytTov ${ }^{1}$ and Francesco SAnnino ${ }^{2}$ \\ The Niels Bohr Institute, Blegdamsvej 17, Copenhagen Ø, Denmark.
}

\begin{abstract}
The 't Hooft and Corrigan-Ramond limits of massless one-flavor QCD consider the two Weyl fermions to be respectively in the fundamental representation or the two index antisymmetric representation of the gauge group. We introduce a limit in which one of the two Weyl fermions is in the fundamental representation and the other in the two index antisymmetric representation of a generic $\mathrm{SU}(\mathrm{N})$ gauge group. This theory is chiral and to avoid gauge anomalies a more complicated chiral theory is needed. This is the generalized Georgi-Glashow model with one vector like fermion.

We show that there is an interesting phase in which the considered chiral gauge theory, for any N, Higgses via a bilinear condensate: The gauge interactions break spontaneously to ordinary massless one-flavor SU(3) QCD. The additional elementary fermionic matter is uncharged under this $\mathrm{SU}(3)$ gauge theory. It is also seen that when the number of colors reduce to three it is exactly this hidden QCD which is revealed.
\end{abstract}

\footnotetext{
${ }^{1}$ E-mail: ryttov@nbi.dk

${ }^{2}$ E-mail: sannino@nbi.dk
} 


\section{Introduction}

Different limits in gauge theories have been explored in the past with the hope to get a better understanding of strongly interacting gauge dynamics. The large $N$ limit a là 't Hooft [1] and its theoretical success [2] have triggered a good deal of past and recent work in different realms of theoretical physics from field theory to string theory. Modern lattice simulations have recently explored this limit [3, 4] as well as phenomenological studies based on low energy meson-meson scattering amplitudes [5]. One can, however, imagine different limits which for certain values of the theory parameters yield Quantum Chromo Dynamics (QCD). Here and in the following we focus our attention on one-flavor QCD.

Immediately after the initial 't Hooft proposal Corrigan and Ramond (CR) suggested a different limit [6] in which one imagines the quarks to be in the antisymmetric rank-two representation of the underlying gauge group $^{3}$. Clearly for three colors this is the fundamental representation of the gauge group, however at large number of colors the theory is not mapped in the 't Hooft limit. For instance quark loops are suppressed in the 't Hooft limit but not in the CR limit. There are a number of immediate consequences suggested in the $\mathrm{CR}$ work and a decade later investigated by Kiritsis and Papavassiliou [7]. Also very recently the idea that certain sectors of these theories can be mapped in sectors of super Yang-Mills (SYM) has been suggested [8]. Using a supersymmetric inspired effective Lagrangian approach some of the $1 / N$ corrections were investigated in [9]. When adding flavors the phase diagram as a function of the number of flavors and colors has been provided in [10]. Further recent explorations of this limit and its link to SYM can be found in [1] while the reader can find a review of the string theory aspects in [12].

The issue of confinement/deconfinement phase transition at nonzero temperature for the $\mathrm{CR}$ limit has been explored in detail in [13]. Here, one of the present authors has shown the appearance of an alternating pattern as a function of number of colors with respect to the remaining symmetries of the center group. These results must be compared with the 't Hooft limit

\footnotetext{
${ }^{3}$ To be more precise Corrigan and Ramond [6] suggested a generalization of QCD in which some flavors were in the higher dimensional representation and others still in the fundamental representation. They focused the paper on the baryonic properties of this theory and the $\eta^{\prime}$ properties at large $N$, as well as technicolor models with fermions in the two index antisymmetric representation of the gauge group.
} 
often used to infer information about QCD.

New technicolor type theories have recently been constructed which make use of new gauge interactions with fermions in the two index (but symmetric) representation of the gauge group [10, 14]. These theories are not ruled out by the electroweak precision measurements [15].

Since these two limits are very different it would be interesting to explore a third one which is somewhat in between the 't Hooft and CR one. To be more specific one would like to have half of the fermions in the rank-two antisymmetric representation and the other half transforming according to the fundamental representation of the gauge group. If such a theory exists, for three colors it would be identical to one-flavor QCD and for a generic number of colors the theory would be, however, chiral. First we have certain issues to address:

- Can one construct a gauge anomaly free chiral theory which for three colors is vector-like and in particular exactly one-flavor QCD ?

- Even if we find such a theory what can we say either about QCD or this theory?

It turns out that it is possible to construct such a gauge anomaly free chiral theory. It is a particular case of the generalized Georgi-Glashow (gGG) model [16]. In this model we have exactly one fermion in the two index antisymmetric representation and other fermions in the fundamental and antifundamental representation of the gauge group. Special relations between the two kinds of fermions are required to avoid the dangerous gauge anomalies. In Figure 1 we schematically summarize the different limits for QCD. Together with their sister theories of the Bars-Yankielowicz (BY) type [17, in which the fermions with two indices are symmetrized, these chiral gauge theories are known to display a number of interesting possible phases in the infrared. See references [18, 19] for a review of some of these properties and an exhaustive list of references on the gGG and BY models.

In the next section we introduce the gGG model. We show the existence of a phase in the model, for a generic number of colors, in which the gauge group spontaneously breaks exactly to massless one-flavor QCD and a remnant part which does not interact in the deep infrared and is disconnected from QCD. This allows us to make a number of predictions for the chiral gauge theory using our QCD knowledge. We also discuss possible pitfalls and alternative scenarios. We finally conclude in section three. 


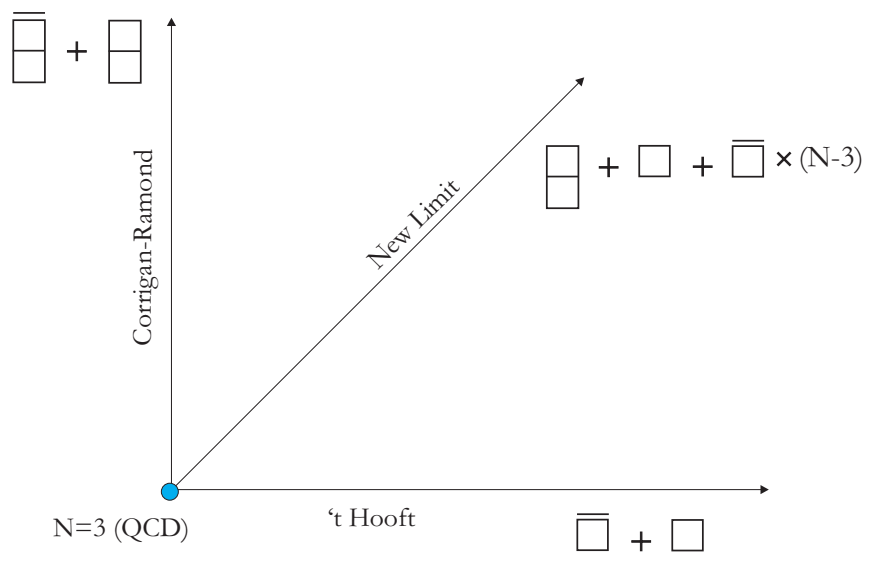

Figure 1: Schematic representation of the matter fields required to construct the three distinct limits which for three colors reduce to massless one-flavor QCD. On each line we increase the number of colors while the gauge group is always $S U(N)$.

\section{The Generalized Georgi-Glashow Model: Hid- den QCD}

The generalized Georgi-Glashow (gGG) model is based on the gauge group $S U(N)$. The matter content of the theory includes fermions $A=\psi_{L}^{[i j]}, i, j=$ $1, \cdots, N$ in the anti-symmetric $\frac{1}{2} N(N-1)$ irreducible tensor representation; $N-4+p$ fermions $\bar{F}_{i, f}=\psi_{i, f L}^{c}, f=1, \cdots, N-4+p$ in the conjugated fundamental representation; and $p$ fermions $F^{i, f}=\psi_{L}^{i, f}, f=1, \cdots, p$ in the fundamental representation as summarized in the table below:

\begin{tabular}{c||ccccc} 
& {$[S U(N)]$} & $S U(N-4+p)$ & $S U(p)$ & $U_{1}(1)$ & $U_{2}(1)$ \\
\hline \hline$A$ & $\boxminus$ & 1 & 1 & $N-4$ & $2 p$ \\
$\bar{F}$ & $\square$ & $\square$ & 1 & $-(N-2)$ & $-p$ \\
$F$ & $\square$ & 1 & $\square$ & $N-2$ & $-(N-p)$
\end{tabular}

The first $S U(N)$ is the gauge group. The two abelian symmetries are anomaly free. They are linear combinations of the original $U(1)$ symmetries 
acting on each fermionic field separately. The antifermion is needed to render the theory free from gauge anomalies.

For now we just note that when $p=1$ and $N=3$ the theory coincides with one-flavor QCD with $F$ being the quark and $A$ being the antiquark.

The beta function of the model is

$$
\beta=\mu \frac{d \alpha}{d \mu}=-\beta_{1}\left(\frac{\alpha^{2}}{2 \pi}\right)-\beta_{2}\left(\frac{\alpha^{3}}{4 \pi^{2}}\right)+O\left(\alpha^{4}\right),
$$

where the terms of order $\alpha^{4}$ and higher are scheme-dependent and $\beta_{1}=$ $3 N+2-(2 / 3) p$ and $\beta_{2}=(1 / 4)\left[13 N^{2}+30 N+1-12 / N-2 p((13 / 3) N-1 / N)\right]$. Thus the theory is asymptotically free if

$$
p<(9 / 2) N+3
$$

There are a number of interesting possibilities studied in the past for the ground state of these theories. The reader can find a list and a guide on how to select among different possible ground states in 19. One of the remarkable properties of these theories is that there are phases in which the whole chiral symmetry does not break and the confining spectrum in the infrared saturates all of the 't Hooft global anomalies of the theory. Besides some of these phases can also be seen as Higgs phases or partial Higgs phases and partial confining. By confining, however, we simply mean that the low energy states are composites of the underlying fermions in a color blind object. Since in these theories the center group symmetry is absent we do not have a good order parameter for the confinement/deconfinement transition. This is similar to the case of one-flavor QCD.

As was noted above the case with $p=1$ is of particular interest. For arbitrary $N$ this is an asymptotically free chiral theory which for $N=3$, coincides with massless one-flavor QCD. It is also clear that in the present limit to QCD the ordinary quark left emerges as in the 't Hooft limit while the conjugated quark right is recovered as in the CR limit. The fermions $\bar{F}$ disappear in the $N=3$ limit. In this sense we consider this theory as a novel limit intermediate with respect to the 't Hooft and CR ones. A straightforward count of the degrees of freedom in the ultraviolet shows that we have $N^{2}$ gluons, $N^{2} \bar{F}$-type fermions and $N^{2} / 2 A$-type fermions. On the other hand in the CR limit we have $N^{2}$ gluons and $N^{2}$ fermions, while in the 't Hooft limit we just have $N^{2}$ gluons. From the QCD point of view we suppress only half of the fermion spectrum and keep the other half. $\bar{F}$ is a fermion needed, for any finite $N$ to ensure that the theory is well defined. 
Although many phases are known and are possible to occur for a given gGG theory still much is unknown about chiral gauge theories per se. This heavily limits our predictive capabilities. However, it is worth mentioning that a large $N$ limit for these theories can be constructed and the expansion organized [20]. So, perhaps, also a string theory description of these theories is possible.

We also notice that these theories have been used in phenomenological models for grand unification, theories of massless composite leptons and quarks and for suggesting purely fermionic gauge theories which might naturally Higgs.

When departing from the three color limit we envision two logical possibilities:

- A phase transition in the number of colors occurs and the chiral gauge theory in the new phase displays a vastly different structure with respect to the three color limit.

- A more subtle phase emerges which allows the theory to smoothly link itself to QCD when the number of colors is three.

The first case may appear in different ways. Here we suggest the following two possibilities. Assume that the gGG theory confines for any number of colors without breaking any of the global symmetries. Then the massless spectrum of this theory must be fermionic and saturate all of the 't Hooft anomaly conditions. This solution does exist and is summarized in the following table:

\begin{tabular}{c||cccc} 
& {$[S U(N)]$} & $S U(N-3)$ & $U_{1}(1)$ & $U_{2}(1)$ \\
\hline \hline $\bar{F} A \bar{F}$ & 1 & $\bar{\square}$ & $-N$ & 0 \\
$\bar{F}^{\dagger} A^{\dagger} F$ & 1 & $\square$ & $N$ & $-N$
\end{tabular}

Here the massless fermionic spectrum is constituted by two types of colorless composite massless bound states, $\bar{F} A \bar{F}$ and $\bar{F}^{\dagger} A^{\dagger} F$. At large number of colors one can drop the fermion $F$ and the only relevant bound state is $\bar{F} A \bar{F}$. The phase transition from three colors to a larger number of colors emerges since massless colorless bound states appear which were not present before. Since the associated flavor symmetries were not present in the QCD case one may still argue that the associated currents simply do not match the 
ones of QCD and that QCD is somewhat hidden in the massive dynamics of this phase. Although we cannot completely exclude this possibility the problem would, in any case, be that within this picture it is hard to see any trace of QCD.

Another possible phase is the one in which the $F$ fermion condenses with $\bar{F}$. In this case the gauge group remains intact while the flavor symmetry breaks to $S U(N-4) \times U_{1}^{\prime}(1) \times U_{1}^{\prime}(1)$ where the last two abelian symmetries are obtained by combining the old abelian symmetries and one of the broken nonabelian generators of the $S U(N-3)$ flavor symmetry. The remaining theory is the one without the $F$ field and can either Higgs or confine (these two cases lead to the same number of massless fermions). Due to the breaking of the flavor symmetry we also have goldstone bosons. The reader can find a review of this phase in [19]. This phase is prohibited for $N$ less than four. We, hence, do not expect this phase to be smoothly connected to the QCD limit.

We will now exhibit an interesting phase in which the theory spontaneously breaks itself into massless one-flavor QCD and elementary massless fermions uncharged under QCD. This can happen for any number of colors. Recall that the full symmetry group of the theory is

$$
[S U(N)] \times S U(N-3) \times U_{1}(1) \times U_{2}(1)
$$

with the first one being the gauge symmetry. Lets consider the following Higgs phase corresponding to the condensation in the attractive channel

$$
\boxminus \times \bar{\square} \rightarrow \square
$$

associated to the formation of the condensate

$$
\left\langle A^{[i j]} \bar{F}_{j, f}\right\rangle
$$

where the spinor indices are contracted and $i, j=1, \ldots, N$ and $f=1, \ldots, N-$ 3. The present channel is more attractive than the $F \bar{F}$ one when the colors are more than four in the one gluon approximation of the potential between the two fermions. It breaks the gauge symmetry from $S U(N)$ to $S U(3)$. Through color-flavor locking the broken gauge subgroup $S U(N-3)$ combines with the flavor group forming a new $S U^{\prime}(N-3)$ global symmetry.

After spontaneous symmetry breaking we still have two abelian symmetries $U_{1}^{\prime}(1) \times U_{2}^{\prime}(1)$ left intact. The first is formed as a linear combination of 
the old abelian symmetries. The second is formed by combining the broken diagonal $S U(N)$ generator

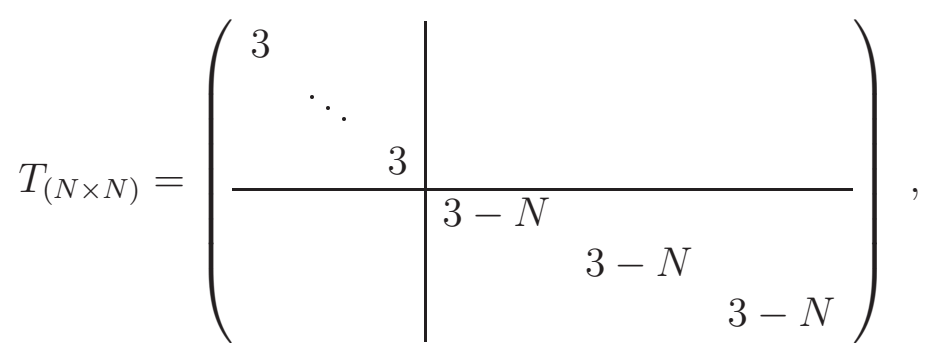

with the old $U_{2}(1)$ symmetry. Thus the pattern of symmetry breaking is

$$
\begin{gathered}
{[S U(N)] \times S U(N-3) \times U_{1}(1) \times U_{2}(1)} \\
\downarrow\langle A \bar{F}\rangle \\
{[S U(3)] \times S U^{\prime}(N-3) \times U_{1}^{\prime}(1) \times U_{2}^{\prime}(1) .}
\end{gathered}
$$

Lets investigate the field content in the spontaneously broken phase. All the Goldstone bosons are eaten up by the massive gauge bosons. The fermions split into

$$
\begin{gathered}
A^{[i j]}=\left(\begin{array}{cc}
A^{\left[f f^{\prime}\right]} & A^{[f c]} \\
A^{[c f]} & A^{\left[c c^{\prime}\right]}
\end{array}\right), \\
\bar{F}_{i, f}=\left(\begin{array}{c}
\bar{F}_{\left[f^{\prime}, f\right]}+\bar{F}_{\left\{f^{\prime}, f\right\}} \\
\bar{F}_{c, f}
\end{array}\right), \\
F^{i}=\left(\begin{array}{c}
F^{f} \\
F^{c}
\end{array}\right)
\end{gathered}
$$

with $f, f^{\prime}=1, \ldots, N-3$ and $c, c^{\prime}=N-2, \ldots, N$. The massless sector of the theory contains $F^{c}$ and $A^{\left[c c^{\prime}\right]}$ which belong to the $\mathbf{3}$ and $\overline{\mathbf{3}}$ of the unbroken gauge subgroup $S U(3)$. We identify them with an ordinary quark and antiquark respectively. Also $F^{f} \equiv L^{f}$ and $\bar{F}_{\left\{f^{\prime}, f\right\}} \equiv \bar{L}_{\left\{f^{\prime}, f\right\}}$ which are color singlets remain massless. Summarizing, the spontaneously broken phase has the following massless fermion content 


\begin{tabular}{c||cccc} 
& {$[S U(3)]$} & $S U^{\prime}(N-3)$ & $U_{1}^{\prime}(1)$ & $U_{2}^{\prime}(1)$ \\
\hline \hline$q$ & $\square$ & 1 & $-N$ & $\frac{2}{3} N$ \\
$\bar{q}$ & $\bar{\square}$ & 1 & $N$ & $-\frac{2}{3} N$ \\
$L$ & 1 & $\square$ & $-N$ & $N$ \\
$\bar{L}$ & 1 & $\bar{\square}$ & $-N$ & 0
\end{tabular}

This shows that for any number of colors we have found a phase of the theory which at low energy splits into massless one-flavor QCD and a number of elementary massless fermions uncharged under the color gauge group. However massless one-flavor QCD confines and gaps as well so that in the deep infrared the only true massless degrees of freedom are indeed the chiral fermions $L$ and $\bar{L}$. Since the global symmetries are unbroken the massless spectrum in the deep infrared, needed to saturate the 't Hooft anomalous conditions, coincides with the one of the confining phase. However the massless spectrum of the theory consists of elementary fermions as opposed to the confining phase which consists of bound states of the original elementary fermions. To observe the physical difference between these different phases one has to go beyond the simple counting of massless degrees of freedom. For instance one should probe the structure of the massless fermions and investigate the massive spectrum of the theory.

In our case we predict different energy ranges: First an energy range above and around the scale $\Lambda$ of the condensate $\langle A \bar{F}\rangle$. Some of the underlying fermions (which can be traced via the analysis performed above) receive mass via this condensate with a mass of the order of $\Lambda$. Except for the massless octet of ordinary gluons all of the remaining gauge bosons will acquire a mass of the order of $g \Lambda \lesssim \Lambda$ where $g$ is the coupling constant evaluated at the energy $\Lambda$. A second energy range is below the scale $\Lambda$ where we have massless one-flavor QCD together with the $L$ and $\bar{L}$ fermions which are uncharged under the QCD gauge group. Then a new scale $\Lambda_{Q C D}<\Lambda$ develops where QCD confines. The third and last energy range is below $\Lambda_{Q C D}$ where only $L$ and $\bar{L}$ survive as massless elementary fermions.

It is very tempting to assume a smooth limit as function of the number of colors near the three color case. If this happens we can predict that the QCD spectrum describes a good part of the massive non-perturbative spectrum of 
the underlying chiral gauge theory. This is so since the Lagrangian splits into two sectors below the scale $\Lambda$ as follows

$\mathcal{L}=\mathcal{L}_{Q C D}(q, \bar{q})+\mathcal{L}_{\text {Non-Gauge }}(L, \bar{L})$. At energy scales smaller than $\Lambda$.(11)

Hence the partition function of the underlying chiral gauge theory factorizes at low energy in the partition function for QCD and the one of low energy massless noninteracting fermions. A part of the massive spectrum of the gGG model corresponds to the one of one-flavor QCD for any number of colors. Surprisingly this phase is now smoothly connected to pure massless one-flavor QCD when the number of colors is three even though our theory is a complicated chiral gauge theory.

When the number of colors is very large the $A$ and $\bar{F}$ fermions dominate the action. This is so since the number of these fermions increases quadratically with the number of colors while the $F$ fermions increase only linearly with respect to the number of colors. Since QCD emerges, in our picture, as a mismatch between the $N$ colors and the $N-3$ flavors we expect this phenomenon to appear only when considering subleading terms in the $1 / N$ expansion of the full theory.

\section{Conclusions}

We have introduced a new limit for massless one-flavor QCD. For three colors one can imagine one of the two Weyl fermions of one-flavor massless QCD to belong to the fundamental representation of the gauge group and the other in the two index antisymmetric representation. When the number of colors is larger than three the theory is chiral. To avoid gauge anomalies a more complicated theory is needed. This is the generalized Georgi-Glashow model with one vector like fermion. While a large number of possible phases for these theories have been constructed, unfortunately very little is known about the ground state of these type of gauge theories and/or the spectrum of massive states. It is possible that when the number of colors increases a possible link with QCD is completely lost. We have shown how this could happen by providing different phases in which this theory would not display any QCD property.

Somewhat surprisingly, we have discovered a phase in which, for any $N$, if we assume the $A \bar{F}$ bilinear to condense, (here we are considering the case of $p=1$ ) the gauge interaction breaks spontaneously to ordinary massless 
one-flavor $S U(3)$ QCD. The additional elementary fermionic matter is uncharged under this $S U(3)$ gauge theory. We have already stressed that such a condensation pattern is partially supported by the fact that the condensate corresponds to a maximally attractive channel when the number of colors is sufficiently large. Besides, when the number of colors reduce to three it is exactly this hidden QCD which is revealed. The two sectors of the theory are disconnected at low energy and we predict the one-flavor QCD spectrum to constitute a relevant part of this certain class of generalized Georgi-Glashow models. The large number of colors limit enhances only half of the QCD fermionic matter. We expect that our limit can be used also when exploring supersymmetric chiral gauge theories.

Together with the 't Hooft and Corrigan-Ramond limits, the present one exhausts all of the possible ways (representation wise) one can envision extending massless one-flavor QCD.

\section{Acknowledgments}

We thank S. Bolognesi, D.D. Dietrich, P. Di Vecchia, M. Frandsen, C. Kouvaris, R. Marotta, M. Shifman, B. Svetitsky and K. Tuominen for discussions and comments.

TR wishes to thank the Lørup Fund and A.D. Jackson for financial support during his stay at CERN. FS is supported by the Marie Curie Excellence Grant as team leader under contract MEXT-CT-2004-013510 and by the Danish Research Agency. We also thank the CERN theory division for the kind hospitality during the initial stages of this work.

\section{References}

[1] G. 't Hooft, "A Planar Diagram Theory For Strong Interactions," Nucl. Phys. B 72 (1974) 461.

[2] E. Witten, "Baryons In The 1/N Expansion," Nucl. Phys. B 160, 57 (1979).

[3] B. Bringoltz and M. Teper, "The pressure of the SU(N) lattice gauge theory at large-N," arXiv:hep-lat/0506034. 
[4] R. Narayanan and H. Neuberger, "The quark mass dependence of the pion mass at infinite N," Phys. Lett. B 616, 76 (2005) arXiv:hep-lat/0503033.

[5] M. Harada, F. Sannino and J. Schechter, "Large N(c) and chiral dynamics," Phys. Rev. D 69, 034005 (2004) arXiv:hep-ph/0309206.

[6] E. Corrigan and P. Ramond, "A Note On The Quark Content Of Large Color Groups," Phys. Lett. B 87, 73 (1979).

[7] E. B. Kiritsis and J. Papavassiliou, "An Alternative Large N Limit For QCD And Its Implications For Low-Energy Nuclear Phenomena," Phys. Rev. D 42, 4238 (1990).

[8] A. Armoni, M. Shifman and G. Veneziano, 'Exact results in nonsupersymmetric large N orientifold field theories," Nucl. Phys. B 667, 170 (2003) arXiv:hep-th/0302163. A. Armoni, M. Shifman and G. Veneziano, "SUSY relics in one-flavor QCD from a new $1 / \mathrm{N}$ expansion," Phys. Rev. Lett. 91 (2003) 191601, arXiv:hep-th/0307097|.

[9] F. Sannino and M. Shifman, "Effective Lagrangians for orientifold theories," Phys. Rev. D 69, 125004 (2004) arXiv:hep-th/0309252.

[10] F. Sannino and K. Tuominen, "Orientifold Theory Dynamics and Symmetry Breaking," Phys. Rev. D 71, 051901 (2005) arXiv:hep-ph/0405209.

[11] J. L. F. Barbon and C. Hoyos, "Small volume expansion of almost supersymmetric large N theories," arXiv:hep-th/0507267.

[12] P. Di Vecchia, A. Liccardo, R. Marotta and F. Pezzella, "The gauge / gravity correspondence for non-supersymmetric theories," Fortsch. Phys. 53, 450 (2005) arXiv:hep-th/0412234.

[13] F. Sannino, "Higher representations: Confinement and large N," arXiv:hep-th/0507251

[14] D. K. Hong, S. D. H. Hsu and F. Sannino, "Composite Higgs from higher representations," Phys. Lett. B 597, 89 (2004) arXiv:hep-ph/0406200. 
[15] D. D. Dietrich, F. Sannino and K. Tuominen, "Light composite Higgs from higher representations versus electroweak precision measurements: Predictions for LHC," arXiv:hep-ph/0505059. To Appear in Phys. Review D.

[16] H. Georgi, "A Tool Kit For Builders Of Composite Models," Nucl. Phys. B 266 (1986) 274.

[17] I. Bars and S. Yankielowicz, "Composite Quarks And Leptons As Solutions Of Anomaly Constraints," Phys. Lett. B 101 (1981) 159.

[18] M. E. Peskin, "Chiral Symmetry And Chiral Symmetry Breaking," SLAC-PUB-3021 Lectures presented at the Summer School on Recent Developments in Quantum Field Theory and Statistical Mechanics, Les Houches, France, Aug 2 - Sep 10, 1982

[19] T. Appelquist, Z. y. Duan and F. Sannino, "Phases of chiral gauge theories," Phys. Rev. D 61, 125009 (2000) arXiv:hep-ph/0001043.

[20] E. Eichten, R. D. Peccei, J. Preskill and D. Zeppenfeld, "Chiral Gauge Theories In The 1/N Expansion," Nucl. Phys. B 268, 161 (1986). 